\section{(1)}

CrossMark

\title{
Pathological mucus and impaired mucus clearance in cystic fibrosis patients result from increased concentration, not altered $\mathrm{pH}$
}

\author{
David B. Hill ${ }^{1,2}$, Robert F. Long², William J. Kissner², Eyad Atieh², \\ Ian C. Garbarine ${ }^{2}$, Matthew R. Markovetz², Nicholas C. Fontana², \\ Matthew Christy ${ }^{2}$, Mehdi Habibpour ${ }^{2}$, Robert Tarran ${ }^{2,3}$, M. Gregory Forest (1) 4,5,6, \\ Richard C. Boucher ${ }^{2}$ and Brian Button $2,6,7$
}

Affiliations: 'Dept of Physics and Astronomy, The University of North Carolina at Chapel Hill, Chapel Hill, NC. USA. ${ }^{2}$ Marsico Lung Institute/CF Center, The University of North Carolina at Chapel Hill, Chapel Hill, NC, USA. ${ }^{3}$ Dept of Cell Biology and Physiology, The University of North Carolina at Chapel Hill, Chapel Hill, NC, USA. ${ }^{4}$ Dept of Mathematics, The University of North Carolina at Chapel Hill, Chapel Hill, NC, USA. ${ }^{5}$ Dept of Applied Physical Sciences, The University of North Carolina at Chapel Hill, Chapel Hill, NC, USA. ${ }^{6}$ Dept of Biomedical Engineering, The University of North Carolina at Chapel Hill, Chapel Hill, NC, USA. ${ }^{7}$ Dept of Biophysics and Biochemistry, The University of North Carolina at Chapel Hill, Chapel Hill, NC, USA.

Correspondence: David B. Hill, Dept of Physics and Astronomy, The University of North Carolina at Chapel Hill, 7109 Marsico Hall, 125 Mason Farms Drive, Chapel Hill, NC 27599, USA. E-mail: dbhillamed.unc.edu

@ERSpublications

Over length scales greater than the mesh size of the mucin polymeric network of mucus, concentration, and not $\mathrm{pH}$, dominates the physical properties of mucus that are associated with cystic fibrosis airways disease http://ow.ly/K92u30m2RDC

Cite this article as: Hill DB, Long RF, Kissner WJ, et al. Pathological mucus and impaired mucus clearance in cystic fibrosis patients result from increased concentration, not altered pH. Eur Respir J 2018; 52: 1801297 [https://doi.org/10.1183/13993003.01297-2018].

ABSTRACT Cystic fibrosis (CF) is a recessive genetic disease that is characterised by airway mucus plugging and reduced mucus clearance. There are currently alternative hypotheses that attempt to describe the abnormally viscous and elastic mucus that is a hallmark of CF airways disease, including: 1) loss of CF transmembrane regulator (CFTR)-dependent airway surface volume (water) secretion, producing mucus hyperconcentration-dependent increased viscosity, and 2) impaired bicarbonate secretion by CFTR, producing acidification of airway surfaces and increased mucus viscosity.

A series of experiments was conducted to determine the contributions of mucus concentration versus $\mathrm{pH}$ to the rheological properties of airway mucus across length scales from the nanoscopic to macroscopic.

For length scales greater than the nanoscopic, i.e. those relevant to mucociliary clearance, the effect of mucus concentration dominated over the effect of airway acidification.

Mucus hydration and chemical reduction of disulfide bonds that connect mucin monomers are more promising therapeutic approaches than alkalisation. 


\section{Introduction}

Airway surface liquid (ASL) protects the lung from airborne environmental irritants and infections. The ASL consists of the periciliary layer (PCL) and mucus layer. The PCL is a grafted polymeric brush of tethered mucins and other high-molecular-weight glycoproteins that provides: 1) lubrication for ciliary beat and mucus layer transport, and 2) a size exclusion barrier [1]. The mucus layer is a viscoelastic fluid comprised of over 1000 different proteins [2], including two secreted mucins, MUC5B and MUC5AC [3], which give mucus its gel-like properties [3]. Collectively, the two layers of the ASL coordinate to trap and clear inhaled pathogens from the lung via mucociliary transport (MCT).

It is well established that cystic fibrosis (CF) airway mucus has abnormal rheological properties, including viscosities and elasticities orders of magnitude higher than normal mucus [1,4-10]. There are currently alternative hypotheses that describe the genesis of the pathologically viscoelastic mucus in CF. One hypothesis predicts that impaired anion transport by the CF transmembrane regulator (CFTR), coupled to persistent sodium absorption, reduces airway surface ion content [5]. The reduction of sodium $\left(\mathrm{Na}^{+}\right)$and chloride $\left(\mathrm{Cl}^{-}\right)$ions on airway surfaces is coupled to reduced water flow to the airway lumen, dehydrating the ASL, concentrating the mucus layer, and producing abnormal mucus viscoelastic properties and osmotic pressures [1, 11]. A competing hypothesis posits that defective CFTR fails to transport bicarbonate $\left(\mathrm{HCO}_{3}^{-}\right)$to the airway surface, acidifying the ASL. The resultant reduced mucus layer $\mathrm{pH}$ is predicted to perturb the rheological properties of the mucus layer and impair clearance [12-14].

Mucus is a soft hydrogel composed of high-molecular-weight mucin polymers in an isotonic solvent. The concentration and molecular weight of mucins in the mucus layer define the average distance between adjacent mucin polymers in solution, i.e. the correlation length. The evidence that concentration profoundly affects airway mucus biophysical properties has emerged from recent measurements of mucus osmotic pressures and microbead rheology that employed probes with diameters between $200 \mathrm{~nm}$ and $2 \mu \mathrm{m}[1,8,11,15]$. The evidence that $\mathrm{pH}$ has effects on CF mucus gel viscosity arose from fluorescence recovery after photobleaching (FRAP) mucus studies that employed probes $<10 \mathrm{~nm}$ in diameter [13]. Due to the length scale-dependent rheological properties of mucus [16-18], it is impossible to compare mucus viscosity measurements directly with probes of differing size. The 5-nm nanoscopic probes employed in FRAP protocols [13] diffuse in the solvent space between mucin molecules, while the larger particles used in microbead rheology assays probe the polymeric mucin network. We hypothesised that the mucus macroscopic rheological properties would correlate better with MCT than nanoscopic measures.

Accordingly, studies were performed to measure the effects of mucus concentration versus $\mathrm{pH}$ on mucus properties and transport at different length scales. Studies of the buffer capacity of mucus as a function of concentration were first performed (see supplementary material). Next, $\mathrm{pH}$ - and mucus concentrationdependent mucus viscosities were measured by FRAP. Biophysical measurements with length scales relevant to mucus gels, e.g. traditional microrheology and microbead rheology with particles larger than the mucus correlation lengths, were performed for comparison with FRAP measurements. Model porcine gastric mucin (PGM), bovine submaxillary mucin (BSM), and human bronchial epithelial (HBE) mucus from both normal and CF cultures were utilised for studies exploring: 1) $\mathrm{pH}$ ranges that exceeded those of normal and CF airway mucus, and 2) mucus concentrations that spanned normal versus CF mucus. The effects of changes in $\mathrm{pH}$ versus concentration on HBE MCT rates were directly compared. To test the relevance of in vitro mucus to disease samples, the concentrations, $\mathrm{pHs}$ and viscoelastic properties of $\mathrm{CF}$ sputum were measured and correlations analysed. Finally, the efficacy of three therapeutic strategies designed to restore mucus viscoelastic properties, e.g. mucus hydration, alkalisation and chemical reduction (i.e. mucolytics), were compared.

\section{Methods}

Mucus samples were prepared as described previously [19, 20], with buffer capacity measurements performed as described elsewhere [21-23]. Spontaneous sputum samples were collected as detailed previously $[11,24]$. Detailed methods for the pooling of sputum samples are given in the supplementary material. Mucus nanorheology was performed with FRAP assays [24], microrheology assays were performed with particle-tracking microrheology of $1-\mu \mathrm{m}$ diameter $\mathrm{COOH}$-modified beads [8] and cone-and-plate macroscopic rheology was performed as described previously [7, 25], ensuring that measurements were taken in the linear viscoelastic regime (supplementary material). Osmotic pressure, PCL height and MCT measurements were performed as described previously [1, 26]. HBE cell cultures and mucus harvested from HBE cell cultures are considered nonhuman subjects under the North Carolina Office of Research Ethics Biomedical Institutional Review Board protocol 03-1396; sputum samples were collected under protocol 15-2431. See supplemental material for detailed methods. 


\section{Results}

\section{Biophysical measurements}

\section{Fluorescence recovery after photobleaching}

Biophysical measurements of complex fluids such as mucus must be tailored to the relevant biological questions (see supplementary material for more details). FRAP was measured using $\sim 5-\mathrm{nm}$ dextran molecules in HBE mucus and CF sputum of varying concentrations to determine the nanoscopic, interstitial rheology of mucus (figure 1a). In both sample types, the diffusion of small molecules was linearly dependent on the concentration of the mucus gels at a constant $\mathrm{pH}$ of 7.2 (figure 1b). ANCOVA performed on the regression lines for $\tau$ mucus $/ \tau$ saline (which equates to the ratio of the viscosity of the sample divide by the viscosity of saline) versus \% solids in HBE mucus and CF sputum revealed that each sample type exhibited significant $(\mathrm{p}<0.01)$ and similar $(\mathrm{p}=0.4$, nonsignificant $)$ correlations with mucus concentration ( $n=3$ per concentration). These data indicate that HBE mucus is a reliable surrogate for the study of concentration-dependent FRAP-measured viscosity of CF sputum.

A second group of experiments examined the effect of $\mathrm{pH}$ on FRAP-measured viscosity in HBE mucus and CF sputum at a constant concentration. Little evidence for a carbon dioxide $\left(\mathrm{CO}_{2}\right)$ tension-dependent effect on FRAP-measured viscosity was observed in HBE mucus $(n=14)$ (figure 1c). In raw CF sputum (9\% solids), a trend towards an increase in FRAP recovery times (proxy for increased viscosity on the nanometre length scale) was observed with acidification produced by $15 \% \mathrm{CO}_{2}(\mathrm{p}=0.19 ; \mathrm{n}=7$ ) (figure 1d). To distinguish whether the $\mathrm{pH}$ effect on FRAP reflected an effect of the solvent or the mucin polymer matrix, the sputum was filtered to remove the mucin polymer matrix (supplementary material). The filtered sputum supernatant exhibited a significant $(\mathrm{p}=0.012 ; \mathrm{n}=9)$ increase in FRAP recovery times at $15 \% \mathrm{CO}_{2}$, similar in pattern to whole sputum (figure 1e). Thus, the 5-nm FRAP probe reported viscosity of the solvent component of mucus, not of the polymeric gel. From the comparison of the composition of HBE mucus and CF sputum, our results indicate that inflammatory proteins generated in vivo in $\mathrm{CF}$ subjects, which are absent in HBE mucus [2], may be responsible for the observed $\mathrm{pH}$-dependent changes in viscosity.

a)

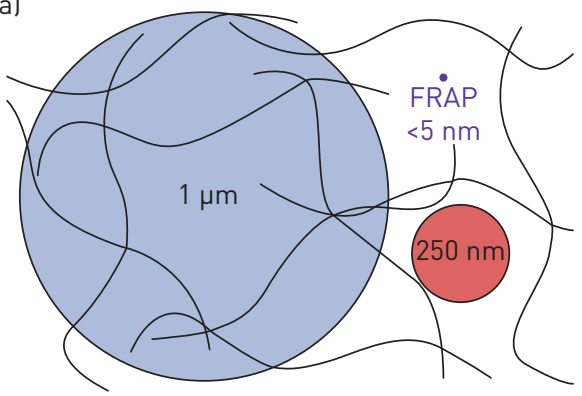

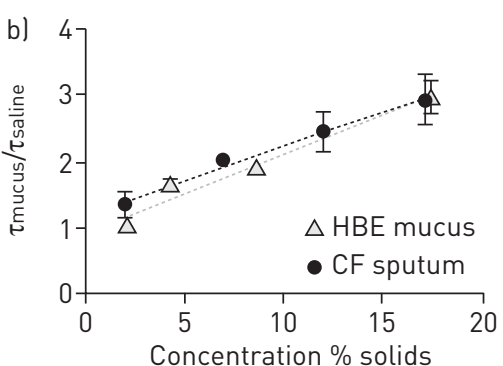

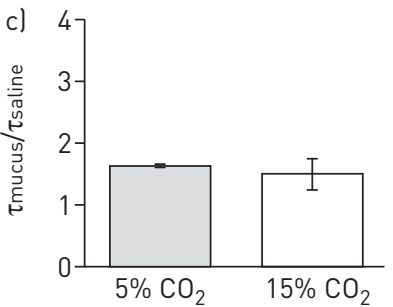

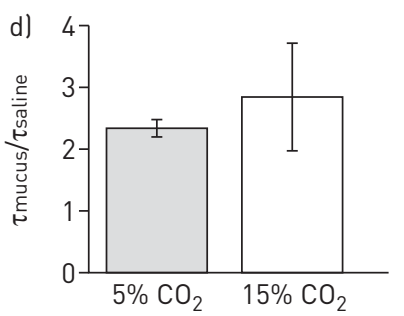

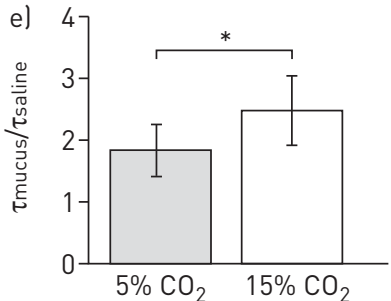

FIGURE 1 Nanoscopic viscosity from fluorescence recovery after photobleaching (FRAP). a) Relevant biophysical size scales in mucus, showing mucin polymers (black lines) with an average correlation length, or mesh size, of $300-500 \mathrm{~nm}$. The 1- $\mu \mathrm{m}$ diameter beads (blue) are larger than the correlation length. The 250-nm beads (red) are sufficiently near to the mesh size that their diffusive motion reflects both the polymeric mucin network as well as the small proteins and solvent that permeate the intra-mucin space. The 5-nm probes (purple) that are typically used in FRAP assays report the rheological properties of the solvent/small-protein component of mucus. b) FRAP recovery times ( $\tau$ mucus/ $\tau$ saline) for human bronchial epithelial (HBE) mucus and cystic fibrosis (CF) sputum at various concentrations ranging from $2 \%$ to $17 \%$ solids. c) FRAP recovery times ( $\tau$ mucus/ $\tau$ saline) for $\mathrm{HBE}$ mucus at $5 \%$ and $15 \% \mathrm{CO}_{2}$, resulting in a change in $\mathrm{pH}$ from 7.2 to 6.6. Nonsignificant, $\mathrm{p}=0.6$. d) FRAP recovery times ( $\tau$ mucus/ $\tau$ saline) for whole, raw sputum at $5 \%$ and $15 \% \mathrm{CO}_{2}$, resulting in a change in $\mathrm{pH}$ from 7.2 to 6.6. Nonsignificant, $\mathrm{p}=0.19$. e) FRAP recovery times ( $\tau$ mucus $/ \tau$ saline) for the filtered, mucin-less solvent component of sputum at $5 \%$ and $15 \% \mathrm{CO}_{2}$, resulting in a change in $\mathrm{pH}$ from 7.2 to $6.6 .^{*}: \mathrm{p}=0.012$. 


\section{Microbead rheology}

Microbead rheology is a well-established technique capable of probing length scales of polymer networks like mucus gels [18]. In PGM studies, a 2.5-fold increase in mucin concentration produced a 5-fold reduction in bead mean squared displacement (MSD) at $1 \mathrm{~s}$ at each designated $\mathrm{pH}$ (figure 2a). In contrast, changes in $\mathrm{pH}$ over a 100 -fold range ( $\mathrm{pH}$ 6-8) produced only a modest (1.5-fold) effect on MSD at $1 \mathrm{~s}$ (figure 2a) at either mucin concentration. Parallel studies of the rheology of BSM showed similar results (figure 2b). Notably, HBE mucus exhibited the greatest dependence on concentration and the least on $\mathrm{pH}$ (figure $2 \mathrm{c}$ ). The MSD of $1-\mu \mathrm{m}$ beads embedded in normal HBE mucus exhibited a 10 times reduction in MSD in response to a 2.5-fold increase in concentration compared with a 1.5 times reduction in MSD in response to a 100-fold increase in proton concentration. A nearly identical result was observed in CF HBE mucus (figure 2d). Two-way ANOVA analysis of the MSD at $1 \mathrm{~s}$ revealed a highly significant relationship between microbead rheology with respect to concentration $(\mathrm{p}<0.01$ for all samples; $n=3$ per mucus type), while statistical significance was absent over the $\mathrm{pH}$ range investigated $(\mathrm{p}>0.05)$

\section{Macroscopic rheology}

The macroscopic, or bulk, rheological properties of mucus are most often related to clearance of mucus from the lung [27-29]. Both concentration and $\mathrm{pH}$ have been shown to affect the rheological properties of mucus at these largest length scales [20,30-32]. Figure 3 shows the relative effects of mucus concentration versus $\mathrm{pH}$ on the complex viscosity of PGM, BSM and HBE mucus. PGM at $50 \mathrm{mg} \cdot \mathrm{mL}^{-1}$ exhibited a viscosity that was $>10$-fold higher than at $20 \mathrm{mg} \cdot \mathrm{mL}^{-1}$. In contrast, $\mathrm{PGM}$ at $20 \mathrm{mg} \cdot \mathrm{mL}^{-1}$ exhibited a complex viscosity that did not vary systematically between $\mathrm{pH} 6$ and 8 (figure 3a). BSM also exhibited large concentration-dependent viscosities, e.g. 20 versus $50 \mathrm{mg} \cdot \mathrm{mL}^{-1}$, with little $\mathrm{pH}$ dependence (figure $3 \mathrm{~b}$ ). With respect to airway mucus, the complex viscosity of $4 \%$ solids HBE mucus was 6- to 10 -fold higher than $2 \%$ solids HBE mucus with again little systematic $\mathrm{pH}$ dependence (figure 3c). A similar pattern was also found in CF HBE mucus (figure 3d). Two-way ANOVA revealed that concentration significantly altered the macroscopic rheology of all mucus types $(\mathrm{p}<0.05)$, while $\mathrm{pH}$ showed no effect ( $p>>0.05 ; n=3$ per mucus type, concentration and $\mathrm{pH}$ ). Thus, the result across all mucus samples was that varying the concentration of mucus altered the complex viscosity dramatically, whereas variations in $\mathrm{pH}$
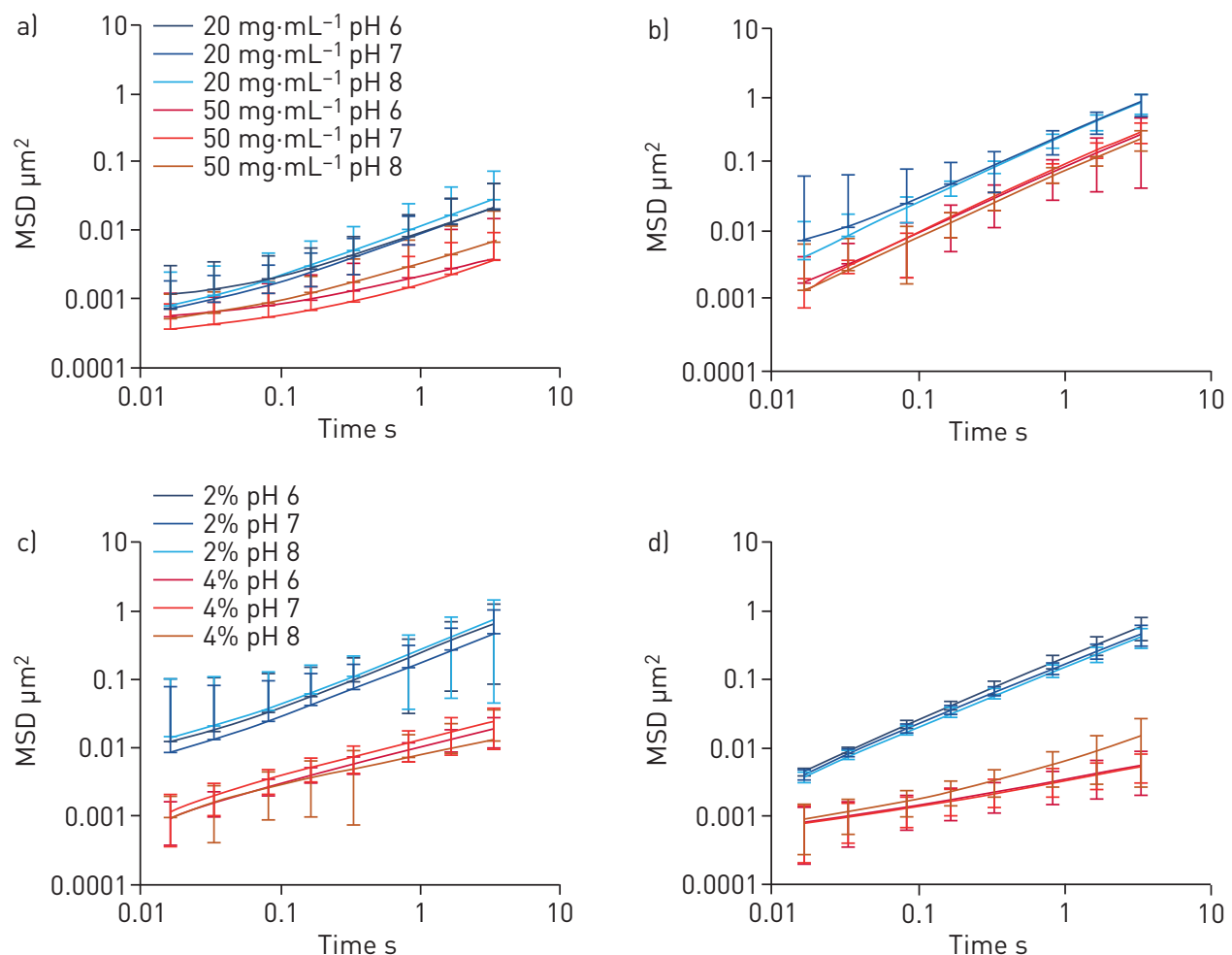

FIGURE 2 Microbead rheology. a, b) Mean squared displacement (MSD) curves for al porcine gastric mucin and b) bovine submaxillary mucin at 20 and $50 \mathrm{mg} \cdot \mathrm{mL}^{-1}$ at $\mathrm{pH} 6,7$ and 8 . c, d) MSD curves for c) human bronchial epithelial (HBE) mucus and d) cystic fibrosis HBE mucus at $2 \%$ and $4 \%$ solids at $\mathrm{pH} 6,7$ and 8 . In each case, the results of two-way ANOVA showed significant dependence of MSD on concentration $(p<0.01)$ and no significance on $\mathrm{pH}(\mathrm{p}>0.05)$. 

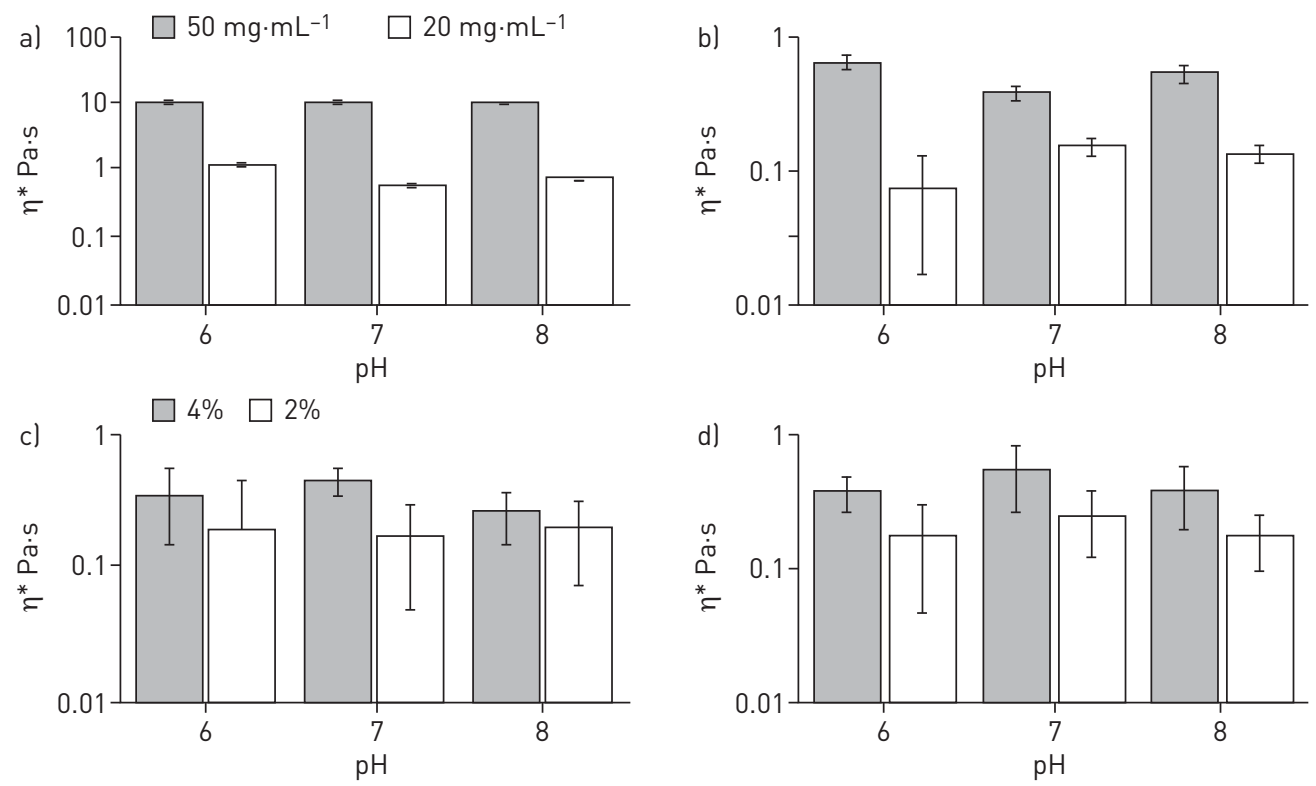

FIGURE 3 Macroscopic rheology of two concentrations of mucus at $\mathrm{pH}$ 6, 7 and 8. a, b) Complex viscosity ( $\eta^{*}$ ) of a) porcine gastric mucin (PGM) and b) bovine submaxillary mucin (BSM) at 20 and $50 \mathrm{mg} \cdot \mathrm{mL}^{-1}$. c, d) Complex viscosity of c) human bronchial epithelial (HBE) mucus and d) cystic fibrosis (CF) HBE mucus at $2 \%$ and $4 \%$ solids. Two-way ANOVA showed that the complex viscosities of PGM and normal and CF HBE mucus were significantly dependent on concentration ( $p=0.031, p=0.038$ and $p=0.020$, respectively), while BSM was of borderline significance $(p=0.05)$. In no case was there a significant correlation between $\mathrm{pH}$ and complex viscosity ( $p=0.46$ for PGM, $p=0.77$ for BSM and $p=0.83$ for HBE mucus). For reference, the viscosity of water is $\sim 0.001 \mathrm{~Pa} \cdot \mathrm{s}$.

did not. It is noteworthy that it is only at $\mathrm{pH}<5$ that significant changes in the viscosity of $20 \mathrm{mg} \cdot \mathrm{mL}^{-1}$ PGM mucus were observed, consistent with the previous findings of CelLi et al. [20] (supplementary material).

\section{Osmotic pressure and PCL height}

The airway surface is lined by two apposing gel layers: 1) a mucus layer containing the secreted MUC5AC and MUC5B mucins, and 2) a brush-like PCL containing tethered mucin (e.g. MUC1, MUC4 and MUC16) and other glycoconjugates [1]. We have previously reported a HBE mucus concentration/osmotic pressure-dependent reduction in PCL height and MCT [24]. We selected a mucus concentration for study that produced a reduction in PCL height to test whether mucus osmotic pressure and PCL height reduction were modified by $\mathrm{pH}$. Changing mucus $\mathrm{pH}$ over a range from $\mathrm{pH} 8$ to 6 did not alter osmotic pressure of a concentrated ( $6.5 \%$ solids) mucus layer $(\mathrm{p} \geqslant 0.14$ between each $\mathrm{pH}$ by paired t-test) (figure $4 \mathrm{a})$. Consistent with the absence of a $\mathrm{pH}$ effect on osmotic pressure, PCL height was also not affected by varying $\mathrm{pH}$ over this range $(\mathrm{p} \geqslant 0.11$ between each $\mathrm{pH}$ by paired $\mathrm{t}$-test) (figure $4 \mathrm{a})$. Note, PCL height with normal mucus concentrations is $\sim 7 \mu \mathrm{m}$.

\section{Mucociliary transport}

MCT measures the integrated activities of cilia beat and mucus properties. Measurements of MCT in HBE cultures identified that the baseline rate of MCT in these cultures over the initial portion of the experiment was $\sim 60 \mu \mathrm{m} \cdot \mathrm{s}^{-1}$. A representative experiment demonstrated that increasing the concentration of $\mathrm{CO}_{2}$ from $5 \%$ to $15 \%$ in the luminal environment produced a decrease in the $\mathrm{pH}$ of the mucus layer from 7.2 to 6.6 over $5 \mathrm{~min}$ (figure $4 \mathrm{~b}$ ). However, this reduction in $\mathrm{pH}$ did not produce a significant change in mucus transport velocity. In contrast, when the concentration of mucus was raised from $2.8 \%$ to $12.6 \%$ by removal of humidity from the environmental chamber, MCT ceased. The mean values for the effect of $\mathrm{pH}$ versus concentration on MCT (figure 4c) demonstrated, as with micro- and macrorheology measurements, mucus concentration $(\mathrm{p}<<0.05)$, not $\mathrm{pH}(\mathrm{p}>>0.05 ; \mathrm{n}=4-12)$, dominated MCT rates.

Relationships between mucus concentration, pH and viscoelastic properties in CF sputum The concentration, $\mathrm{pH}$ and rheology of $11 \mathrm{CF}$ sputum samples were measured (table 1). The average concentration of these samples was $5.6 \pm 2.5 \%$, the average $\mathrm{pH}$ was $7.1 \pm 0.42$ and the average complex viscosity was $2.5 \pm 1.5 \mathrm{~Pa} \cdot \mathrm{s}$. A significant correlation was observed between concentration and complex 

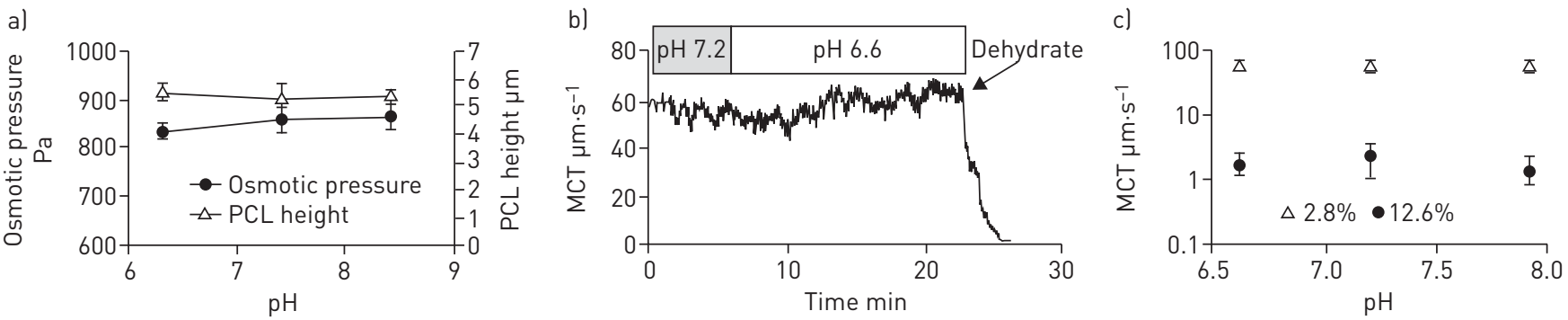

FIGURE 4 Effect of pH on osmotic pressure, periciliary layer (PCL) height and mucociliary transport (MCT). a) Effect of pH on mucus osmotic pressure and PCL height of $6.5 \%$ solids mucus on human bronchial epithelial cell culture. Note that the PCL height is normally $\sim 7 \mu \mathrm{m}$ with a normal $2 \%$ solids $(100 \mathrm{~Pa})$ mucus layer apposed to it. b) Example trace of the effects of acidification $\left(5 \% \mathrm{CO}_{2}, \mathrm{pH} 7.2\right.$ to $\left.15 \% \mathrm{CO}, \mathrm{pH} 6.6\right)$ compared with changes in mucus concentration (by dehumidification) on MCT rate during a single experiment. C) Summary of the MCT rates comparing changes in concentration: $2.8 \%$ or $12.6 \%$ solids versus $\mathrm{pH} 6.6,7.2$ and 7.9 .

viscosity ( $\mathrm{p}=0.006$ ) (figure $5 \mathrm{a}$ ). In contrast, no correlation was observed between $\mathrm{pH}$ and complex viscosity $(\mathrm{p}=0.42)$ (figure $5 \mathrm{~b}$ ). Thus, our data from cultured normal and CF HBE mucus appear to predict data from CF sputum.

\section{Testing therapeutic strategies to normalise CF sputum viscoelastic properties}

To generate sufficient material for systematic studies of therapeutic agents, four sputum samples were pooled and homogenised in the presence of protease inhibitors to blunt proteolysis. This pooled stock was similar in concentration, $\mathrm{pH}$ and rheology to the sample group as a whole (supplementary material). The concentration of the pooled samples (5.2\%) mimicked the average concentration of all CF sputum samples (5.6\%). Aliquots of the pooled sputum were hydrated to $2.6 \%$ to mimic the effect of a mucus hydrator [33, 34 , and then aliquots of sputum at both $2.6 \%$ and $5.2 \%$ concentrations were adjusted to $\mathrm{pH} 6,7$ and 8 to test the effects of modulating $\mathrm{pH}$ on $\mathrm{CF}$ sputum.

Diluting (hydrating) mucus by a factor of 2 from $5.2 \%$ to $2.6 \%$ decreased the complex viscosity by a factor of 8 . In contrast, the effects of a 100 -fold range in $\mathrm{pH}$ were small, i.e. $\sim 2$-fold change in complex viscosity (figure 5c). Importantly, increasing the $\mathrm{pH}$ of $5.2 \% \mathrm{CF}$ sputum from $\mathrm{pH} 6$ to 8 resulted in a complex viscosity greater than the most acidic $2.6 \%$ concentration sputum sample (figure $5 \mathrm{c}$ ). Thus, therapies directed at hydration will likely produce more favourable changes in $\mathrm{CF}$ sputum viscosity than modulation of $\mathrm{pH}$.

Finally, the effect of reduction of mucin disulfide bonds was tested. We chose to reduce mucus with dithiothreitol (DTT) rather than DNase as it has previously been shown to have a greater effect on sputum rheology [35]. Normal HBE mucus was prepared to 5\% solids to mimic CF sputum and reduced with 1 and $10 \mathrm{mM}$ DTT versus PBS control. These data (figure 5d) showed that reduction of the disulfide bonds that link mucin monomers into polymeric macromolecules had a greater effect on the rheological

TABLE 1 Demographics of cystic fibrosis patients from whom the 11 sputum samples were obtained

\begin{tabular}{lc} 
Patients & 11 \\
Sex & \\
$\quad$ Male & 4 \\
$\quad$ Female & 7 \\
Ethnicity & \\
$\quad$ Caucasian & 10 \\
$\quad$ African-American & 1 \\
Age years & $28.5 \pm 9.6(18-46)$ \\
FEV1 \% pred & $48.8 \pm 22.0(24-90)$ \\
FVC L & $2.7 \pm 1.0(1.7-4.3)$ \\
FEF25-75\% L-s & -1 \\
pH & $0.96 \pm 0.82(0.3-2.7)$ \\
Mucus concentration \% solids & $7.1 \pm 0.42(6.3-7.7)$ \\
Complex viscosity $\boldsymbol{\eta}^{*}$ Pa-s & $5.6 \pm 2.5(2.3-10.6)$ \\
\hline
\end{tabular}

Data are presented as $\mathrm{n}$ or mean \pm SD (range). FEV1: forced expiratory volume in $1 \mathrm{~s}$; FVC: forced vital capacity; FEF $25-75 \%$ : forced expiratory flow at $25-75 \%$ of FVC. Spirometry data were obtained for each patient at the time of sample collection. 

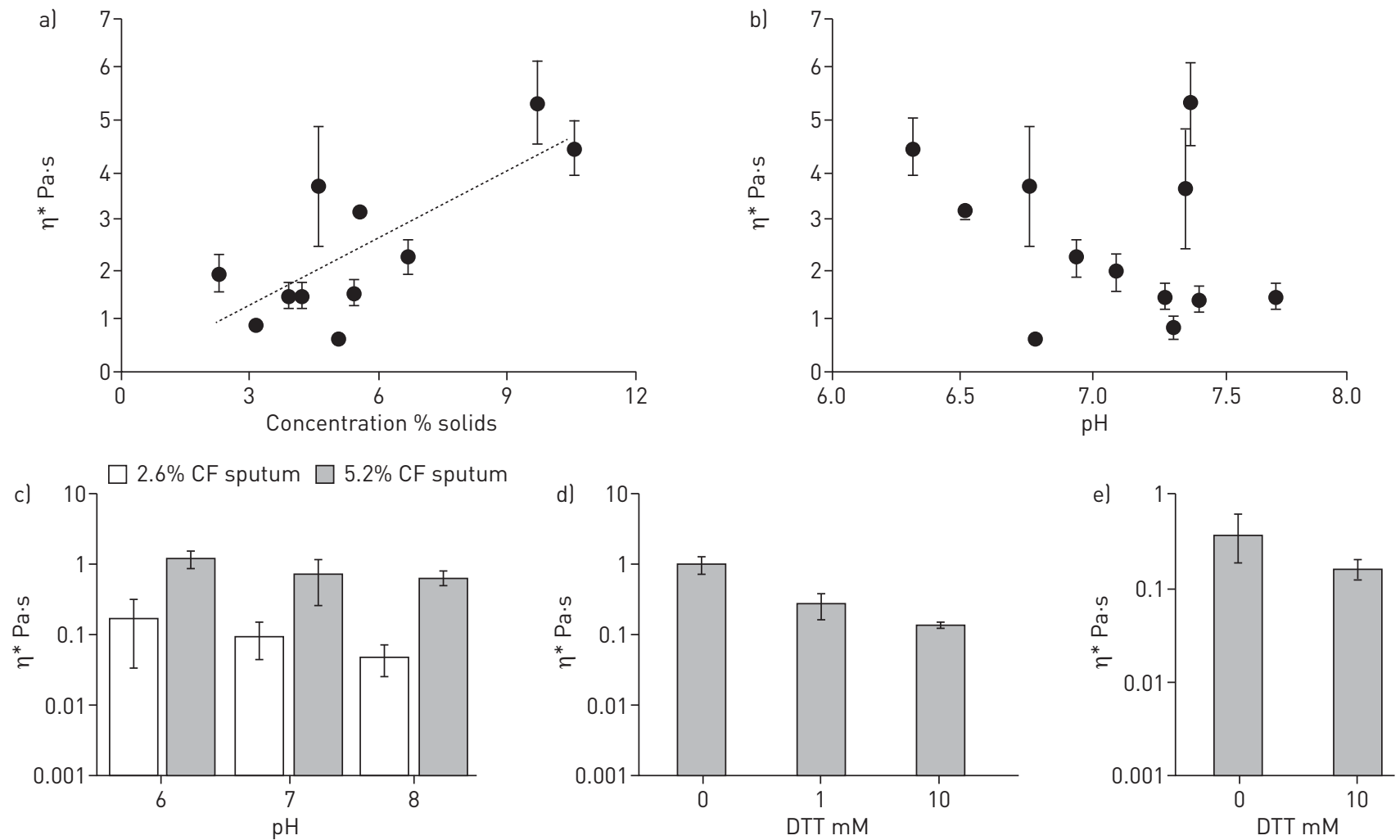

FIGURE 5 Cystic fibrosis (CF) sputum targets and candidate muco-corrective therapeutic strategies. a) Complex viscosity ( ${ }^{*}$ ) versus \% solids for CF sputum exhibited a significant relationship ( $\left.n=11 ; p=0.006, r^{2}=0.58\right)$. b) Complex viscosity versus pH for $\mathrm{CF}$ sputum samples exhibited no significant correlation ( $p=0.42$ ). c) Complex viscosity of pooled, homogenised CF sputum at $2.6 \%$ and $5.2 \%$ solids and $\mathrm{pH} 6,7$ and 8 . Two-way ANOVA indicated that complex viscosity was correlated to concentration ( $p=0.013)$, but not $\mathrm{pH}(\mathrm{p}=0.10)$. d) Chemical reduction of normal human bronchial epithelial mucus by dithiothreitol (DTT). The reduction of the complex viscosity of mucus by $10 \mathrm{mM}$ DTT was found to be significantly different than control $(p=0.027)$. e) Chemical reduction of $5 \%$ pooled CF sputum shows a similar reduction when treated with $10 \mathrm{mM}$ DTT ( $p=0.008$ ). For reference, the viscosity of water is $\sim 0.001 \mathrm{~Pa} \cdot \mathrm{s}$.

properties of mucus than $\mathrm{pH}$, i.e. exhibiting an effect similar to reducing concentration. This result was mimicked by the reduction of $5 \%$ pooled CF sputum with 10 mM DTT (figure 5e).

\section{Discussion}

CF airways disease is characterised by highly viscous airway mucus that leads to mucus stasis, inflammation, chronic infection and damage to airway walls. To test the current hypotheses that may account for the increased viscosity of CF mucus, i.e. mucus acidification [13] versus concentration [1,8, $11,15]$, the relative effects of each variable were measured in model mucus systems (PGM and BSM), both normal and CF HBE mucus, and CF sputum.

The $\mathrm{pH}$ ranges observed in human airway mucus [36,37] are much narrower than in mucus in other organs that are typically studied to describe $\mathrm{pH}$ effects on mucus properties, e.g. the endocervix [32] or stomach [20]. The $\mathrm{pH}$ range of normal human airway mucus is $\mathrm{pH} \sim 7.0-7.2$. It is not clear how much lower the $\mathrm{pH}$ of mucus in $\mathrm{CF}$ airways may be. CF airway epithelial cultures and CF animal models exhibit a $\mathrm{pH}$ that may be $\sim 0.4$ lower than normal airway mucus, i.e. $\sim \mathrm{pH} 6.8[36,38]$. The lowest $\mathrm{pH}$ reported for $\mathrm{CF}$ airway mucus emanated from studies of $\mathrm{pH}$ of mucopurulent material contained in excised $\mathrm{CF}$ lungs and was $\mathrm{pH} 6.6$ [37]. However, recent in vivo data have described little difference in large airway $\mathrm{pH}$ in $\mathrm{CF}$ versus normal neonates [39]. Our strategy to address all possible $\mathrm{CF}$ mucus $\mathrm{pH}$ versus concentration scenarios was to develop protocols that spanned: 1) pH ranges relevant to both $\mathrm{CF}$ ( $\mathrm{pH}$ 6-8) and mucus from other organs ( $\mathrm{pH} 4-8)$, and 2 ) mucus concentrations from normal ( $2 \%$ solids) and CF subjects ( $>5 \%$ solids) [11].

The mucus gel is composed of high-molecular-weight mucin polymers dissolved in an ASL solvent. In the concentrations reported for normal and diseased mucus, the mucin polymers are in "overlap" concentrations, meaning the mucin polymers interpenetrate. Mucin polymers in overlap conditions typically exhibit viscoelastic properties (e.g. viscosity, elasticity [40]) that scale geometrically with 
concentration. The correlation length $\xi$, i.e. mesh size produced by interpenetrating mucins, also scales to a higher-order power of concentration $\left(\xi \sim k T /[\text { concentration] }]^{3}\right)$ and has been reported to be $\sim 100-500 \mathrm{~nm}$ in normal mucus $[15,41]$. The ASL solvent for mucus gels on airway surfaces has been characterised as an isotonic liquid $[42,43]$.

The smallest nanoscopic rheological length scales of mucus scale to its solvent, which can be explored by FRAP. Recent FRAP studies by TANG et al. [13], employing FITC-labelled 70-kDa dextran (radius of gyration $6.6 \mathrm{~nm}$ [44]), demonstrated modest $\mathrm{pH}$-dependent changes in the diffusion of these small molecules in mucus. Our experiments with CF sputum, but not HBE mucus, are in agreement with the FRAP data obtained by TANG et al. [13] in isolated CF piglet mucus (figures 3 and 5). We interpret these data as indicating that the low-molecular-weight (potentially inflammatory) proteins dissolved in the isotonic mucus solvent generated $\mathrm{pH}$-dependent changes in viscosity that exceeded that of water. Notably, the FRAP viscosity values measured in this study $(0.001-0.003 \mathrm{~Pa} \cdot \mathrm{s})$ and those of TANG et al. [13] were orders of magnitude lower than the viscosity values of mucus $(0.1-10 \mathrm{~Pa} \cdot \mathrm{s})$ as measured by macrorheology (see following paragraph and figure 3). The disparity in FRAP versus macrorheology measurements, coupled with the mucin matrix-solvent separation studies (figure $2 b$ ), indicates that the small-molecule FITC-labelled dextrans utilised in FRAP studies measured the $\mathrm{pH}$-sensitive properties of the low-viscosity mucus solvent and not the mucin structure/function that dominates mucus clearance.

Microrheology protocols were performed with $1-\mu \mathrm{m}$ beads, which probe mucus at length scales relevant to the mucin mesh. Our microrheology studies of both PGM and BSM mucus showed a strong (geometric) dependence of mucus complex viscosity on mucus concentration. In contrast, $\mathrm{pH}$ effects on PGM complex viscosity were much smaller and only significant at $\mathrm{pH}<5$ (supplementary material). These findings are in agreement with the data of WANG et al. [32] in MUC5B-dominated endocervical mucus. Importantly, the effects of $\mathrm{pH}$ on $\mathrm{HBE}$ mucus complex viscosity over $\mathrm{pH}$ ranges that span those of airway mucus in health or disease (i.e. $\mathrm{pH}$ 6-8) were minimal compared with the effects of concentration on mucus harvested from both normal and CF cultures.

Conventional cone-and-plate studies probe mucus rheology at macroscopic length scales. With this approach, similar directional and quantitative data were observed as for microrheology (figure 3), i.e. $\mathrm{pH}$ had little effect on complex viscosity over airway $\mathrm{pH}$-relevant ranges. In contrast, strong concentrationdependent relationships were observed, consistent with data of GEORGIADES et al. [31]. Although it has typically been difficult to reconcile findings probing mucus rheological properties at different length scales, our findings at all length scales are consistent with mucus concentration, not $\mathrm{pH}$, dominating mucus mesh-dependent rheological properties relevant to MCT.

Studies of CF sputum were performed for comparison with our mucus model systems. In raw sputum from CF patients, like our model systems, a strong correlation between mucus concentration $(\mathrm{p}<0.05)$, but not $\mathrm{pH}(\mathrm{p}>0.3)$, and rheology was observed (figure $5 \mathrm{a}$ and $\mathrm{b})$.

The water-drawing power of a polymer gel is described by its osmotic pressure. As with rheological properties, osmotic pressures of polymer gels scale to higher-order powers of polymer (mucus) concentration. Under normal conditions, the PCL is the more concentrated gel and is fully hydrated, permitting the cilia to beat in a well-lubricated PCL environment. However, our data have shown that even minor osmotic compression of the PCL by a modestly hyperconcentrated mucus layer (i.e. $4-5 \%$ solids) produces sufficient compression of the cilia to slow mucus transport. Data presented in figure $4 \mathrm{a}$ demonstrate that altering the $\mathrm{pH}$ across acidic versus alkaline ranges did not affect the osmotic properties of the concentrated mucus layer. In contrast, modest mucus hyperconcentration produced the predicted modest PCL compression (figure 4a).

To directly compare the effects of $\mathrm{pH}$ versus concentration on MCT rates, the effects of mucus $\mathrm{pH}$ versus concentration on MCT were measured in HBE cultures (figure $4 \mathrm{~b}$ and c). Consistent with the micro- and macrorheology, MCT was governed by mucus concentration and not $\mathrm{pH}$. These findings are consistent with previous reports that showed MCT is dependent on mucus concentration, with MCT slowing at $4-5 \%$ solids and ceasing at $8-10 \%$ solids $[24,45]$.

Therapeutic strategies to normalise CF mucus biophysical properties focused on concentration (hydration), $\mathrm{pH}$ and disulfide bond reduction. Studies of CF sputum samples exposed to 2-fold variations in concentration versus 100 -fold variations in $\mathrm{pH}$ demonstrated that concentration, not $\mathrm{pH}$, normalised sputum complex viscosity. Chemical reduction of mucus by cleaving disulfide bonds with DTT decreased the complex viscosity of mucus by similar fold values as hydration in both HBE mucus (figure 5d) and pooled CF sputum (figure 5e). These data indicate that both hydration and chemical reduction may be more effective muco-corrective treatments than alkalisation. 
The importance of concentration for the biophysical properties of mucus is rooted in the polymeric nature of the mucin macromolecules that are the key gel-forming molecules in mucus. Increasing the concentration of mucus and mucins in disease states $[8,11,24]$ geometrically alters the viscoelastic and osmotic properties of mucus [40], leading to a collapsed PCL [1], decreased mucociliary clearance [24], reduced neutrophil penetration and bacteria killing [45], biofilm formation [15], and decreased pulmonary function [24, 41, 46]. Collectively, increased mucus concentration can generate much of the muco-obstructive pathophysiology typical of CF $[8,11,24,41]$.

The role of $\mathrm{pH}$ in airway disease states is less clear, with studies differing on the magnitude of the change in $\mathrm{pH}$ across nasal models [47], newborn piglets [13], human cell cultures [36, 38, 39] and early in CF patients in vivo [39]. Our data strongly argue that $\mathrm{pH}$ has little effect on the biophysical properties of mucus relevant to basal mucus transport. It is possible that there is a role for acidification in bacterial killing [48] that may add to failed mucus clearance in CF disease pathogenesis. We speculate, though, that pathophysiologically relevant $\mathrm{CF}$ defects in airway $\mathrm{pH}$ regulation may be more important during gastric aspiration. After aspiration, the $\mathrm{pH}$ of the airway surface is acutely acidified and takes longer to return to baseline in CF than normal cultures [38]. Previous macro- and microscopic rheology studies found that a reduction to $\mathrm{pH}$ 1-3 was required to produce the magnitude of mucus rheological abnormalities produced by CF-relevant increases in mucus concentration [20,30-32]. Thus, gastric aspiration may be combined with increased mucus concentration to trigger early CF events and/or spread of CF lung disease.

\section{Conclusions}

Over the micrometre to millimetre length scales that probe the micro- and macrorheological properties of mucin structure, mucus concentration, not $\mathrm{pH}$, governed the biophysical properties of mucus important for clearance from the lung. In contrast, FRAP studies that probe the solvent properties of the gel are not relevant to the mucus mesh. Data from the larger length scale biophysical assays, but not FRAP, were congruent with data from HBE MCT assays that also demonstrated the dominance of concentration in controlling MCT. Mucus $\mathrm{pH}$ may have effects on antimicrobial killing in the CF lung. However, our findings indicate that therapies designed to restore transport properties of mucus should focus on reducing mucus concentration and/or mucolytic reduction rather than raising $\mathrm{pH}$.

Acknowledgements: The authors wish to thank Erik Perou, Matthew Combs and Jessica Malachowski (all at The University of North Carolina at Chapel Hill, Chapel Hill, NC, USA) for their experimental efforts on this project. The authors would also like to thank Scott Randell and the UNC Tissue Core (The University of North Carolina at Chapel Hill) for HBE cells, and Eric Roe (The University of North Carolina at Chapel Hill) for editorial assistance.

Author contributions: D.B. Hill and B. Button conceived the experiments. D.B. Hill, R.F. Long, R. Tarran, M.G. Forest and B. Button designed the experiments, which were carried out by D.B. Hill, R.F. Long, E. Atieh, I.C. Garbarine, M. Habibpour, N.C. Fontana, W.J. Kissner, M. Christy and B. Button. Data interpretation was performed by D.B. Hill, R.F. Long, M.R. Markovetz, M.G. Forest, R.C. Boucher and B. Button. The manuscript was primarily written by D.B. Hill, R.C. Boucher and B. Button. All authors provided critical feedback that helped shape the manuscript.

Conflict of interest: D.B. Hill reports grants from Cystic Fibrosis Foundation, National Science Foundation and National Institutes of Health, during the conduct of the study. R.F. Long has nothing to disclose. W.J. Kissner reports grants from NIH National Institute of Diabetes, Digestive, and Kidney Diseases (federal), National Science Foundation (federal), KNOW Bio, LLC (business and industry), NIH National Heart, Lung, and Blood Institute (federal), Cystic Fibrosis Foundation (foundation) and Cystic Fibrosis Foundation Therapeutics (foundation), during the conduct of the study. E. Atieh has nothing to disclose. I.C. Garbarine reports grants from Cystic Fibrosis Foundation, during the conduct of the study. M.R. Markovetz has nothing to disclose. N.C. Fontana has nothing to disclose. M. Christy has nothing to disclose. M. Habibpour has nothing to disclose. R. Tarran has nothing to disclose. M.G. Forest has nothing to disclose. R.C. Boucher is chairman of the board of Parion Sciences, a privately held UNC spin-out company focused on developing therapies for $\mathrm{CF}$, and has equity in the company and received monetary compensation as board chair. B. Button has nothing to disclose.

Support statement: This work was funding by the National Science Foundation (DMS 1462992), the National Institutes of Health (R01HL125280, 5P30DK065988 and 4P50HL120100) and the Cystic Fibrosis Foundation (Hill16XX0, Ramsey16I0, Bouche15R0 and Button07XX0). Funding information for this article has been deposited with the Crossref Funder Registry.

\section{References}

1 Button B, Cai LH, Ehre C, et al. A periciliary brush promotes the lung health by separating the mucus layer from airway epithelia. Science 2012; 337: 937-941.

2 Kesimer M, Kirkham S, Pickles RJ, et al. Tracheobronchial air-liquid interface cell culture: a model for innate mucosal defense of the upper airways? Am J Physiol Lung Cell Mol Physiol 2009; 296: L92-L100.

3 Thornton DJ, Rousseau K, McGuckin MA. Structure and function of the polymeric mucins in airways mucus. Annu Rev Physiol 2008; 70: 459-486.

4 Boucher RC. An overview of the pathogenesis of cystic fibrosis lung disease. Adv Drug Deliv Rev 2002; 54: 1359-1371. 
Boucher RC. New concepts of the pathogenesis of cystic fibrosis lung disease. Eur Respir J 2004; 23: 146-158.

Dawson M, Wirtz D, Hanes J. Enhanced viscoelasticity of human cystic fibrotic sputum correlates with increasing microheterogeneity in particle transport. J Biol Chem 2003; 278: 50393-50401.

Hill DB, Button B. Establishment of respiratory air-liquid interface cultures and their use in studying mucin production, secretion, and function. Methods Mol Biol 2012; 842: 245-258.

Hill DB, Vasquez PA, Mellnik J, et al. A biophysical basis for mucus solids concentration as a candidate biomarker for airways disease. PLoS One 2014; 9: e87681.

Rubin BK. Mucus structure and properties in cystic fibrosis. Paediatr Respir Rev 2007; 8: 4-7.

10 Williams HD, Behrends V, Bundy JG, et al. Hypertonic saline therapy in cystic fibrosis: do population shifts caused by the osmotic sensitivity of infecting bacteria explain the effectiveness of this treatment? Front Microbiol 2010; 1: 120 .

11 Henderson AG, Ehre C, Button B, et al. Cystic fibrosis airway secretions exhibit mucin hyperconcentration and increased osmotic pressure. I Clin Invest 2014; 124: 3047-3060.

12 Birket SE, Davis JM, Fernandez CM, et al. Development of an airway mucus defect in the cystic fibrosis rat. JCI Insight 2018; 3: e97199.

13 Tang XX, Ostedgaard LS, Hoegger MJ, et al. Acidic $\mathrm{pH}$ increases airway surface liquid viscosity in cystic fibrosis. J Clin Invest 2016; 126: 879-891.

14 Fischer H, Widdicombe JH. Mechanisms of acid and base secretion by the airway epithelium. J Membr Biol 2006; 211: 139-150.

15 Matsui H, Wagner VE, Hill DB, et al. A physical linkage between cystic fibrosis airway surface dehydration and Pseudomonas aeruginosa biofilms. Proc Natl Acad Sci USA 2006; 103: 18131-18136.

16 Witten J, Ribbeck K. The particle in the spider's web: transport through biological hydrogels. Nanoscale 2017; 9: 8080-8095.

17 Newby JM, Seim I, Lysy M, et al. Technological strategies to estimate and control diffusive passage times through the mucus barrier in mucosal drug delivery. Adv Drug Deliv Rev 2018; 124: 64-81.

18 Lai SK, Wang YY, Wirtz D, et al. Micro- and macrorheology of mucus. Adv Drug Deliv Rev 2009; 61: 86-100.

19 Fulcher ML, Randell SH. Human nasal and tracheo-bronchial respiratory epithelial cell culture. Methods Mol Biol 2013; 945: 109-121.

20 Celli JP, Turner BS, Afdhal NH, et al. Rheology of gastric mucin exhibits a pH-dependent sol-gel transition. Biomacromolecules 2007; 8: 1580-1586.

21 Holma B. Influence of buffer capacity and $\mathrm{pH}$-dependent rheological properties of respiratory mucus on health effects due to acidic pollution. Sci Total Environ 1985; 41: 101-123.

22 Holma B, Hegg PO. pH- and protein-dependent buffer capacity and viscosity of respiratory mucus. Their interrelationships and influence on health. Sci Total Environ 1989; 84: 71-82.

23 Kim D, Liao J, Hanrahan JW. The buffer capacity of airway epithelial secretions. Front Physiol 2014; 5: 188.

24 Anderson $\mathrm{WH}$, Coakley $\mathrm{RD}$, Button $\mathrm{B}$, et al. The relationship of mucus concentration (hydration) to mucus osmotic pressure and transport in chronic bronchitis. Am J Respir Crit Care Med 2015; 192: 182-190.

25 Seagrave J, Albrecht HH, Hill DB, et al. Effects of guaifenesin, $N$-acetylcysteine, and ambroxol on MUC5AC and mucociliary transport in primary differentiated human tracheal-bronchial cells. Respir Res 2012; 13: 98.

26 Button B, Okada SF, Frederick CB, et al. Mechanosensitive ATP release maintains proper mucus hydration of airways. Sci Signal 2013; 6: ra46.

27 Shih CK, Litt M, Khan MA, et al. Effect of nondialyzable solids concentration and viscoelasticity on ciliary transport of tracheal mucus. Am Rev Respir Dis 1977; 115: 989-995.

28 Winet $\mathrm{H}$. The role of the periciliary fluid in mucociliary flows: flow velocity profiles in frog palate mucus. Biorheology 1987; 24: 635-642.

29 Winet H, Yates GT, Wu TY, et al. On the mechanics of mucociliary flows. III. Flow-velocity profiles in frog palate mucus. J Appl Physiol 1984; 56: 785-794.

30 Wagner CE, Turner BS, Rubinstein M, et al. A rheological study of the association and dynamics of MUC5AC gels. Biomacromolecules 2017; 18: 3654-3664.

31 Georgiades P, Pudney PD, Thornton DJ, et al. Particle tracking microrheology of purified gastrointestinal mucins. Biopolymers 2014; 101: 366-377.

32 Wang YY, Lai SK, Ensign LM, et al. The microstructure and bulk rheology of human cervicovaginal mucus are remarkably resistant to changes in pH. Biomacromolecules 2013; 14: 4429-4435.

33 Goralski JL, Wu D, Thelin WR, et al. The in vitro effect of nebulised hypertonic saline on human bronchial epithelium. Eur Respir J 2018; 51: 1702652.

34 Donaldson SH. Hydrator therapies for cystic fibrosis lung disease. Pediatr Pulmonol 2008; 43: S18-S23.

35 Horsley A, Rousseau K, Ridley C, et al. Reassessment of the importance of mucins in determining sputum properties in cystic fibrosis. J Cyst Fibros 2014; 13: 260-266.

36 Coakley RD, Boucher RC. Regulation and functional significance of airway surface liquid pH. JOP 2001; 2: Suppl., 294-300.

37 Garland AL, Walton WG, Coakley RD, et al. Molecular basis for $\mathrm{pH}$-dependent mucosal dehydration in cystic fibrosis airways. Proc Natl Acad Sci USA 2013; 110: 15973-15978.

38 Coakley RD, Grubb BR, Paradiso AM, et al. Abnormal surface liquid pH regulation by cultured cystic fibrosis bronchial epithelium. Proc Natl Acad Sci USA 2003; 100: 16083-16088.

39 Schultz A, Puvvadi R, Borisov SM, et al. Airway surface liquid pH is not acidic in children with cystic fibrosis. Nat Commun 2017; 8: 1409.

40 Rubinstein M, Colby RH. Polymer Physics. Oxford, Oxford University Press, 2003.

41 Duncan GA, Jung J, Joseph A, et al. Microstructural alterations of sputum in cystic fibrosis lung disease. JCI Insight 2016; 1: e88198.

42 Matsui H, Davis CW, Tarran R, et al. Osmotic water permeabilities of cultured, well-differentiated normal and cystic fibrosis airway epithelia. J Clin Invest 2000; 105: 1419-1427.

43 Matsui H, Grubb BR, Tarran R, et al. Evidence for periciliary liquid layer depletion, not abnormal ion composition, in the pathogenesis of cystic fibrosis airways disease. Cell 1998; 95: 1005-1015. 
44 Armstrong J, Wenby R, Meiselman $\mathrm{H}$, et al. The hydrodynamic radii of macromolecules and their effect on red blood cell aggregation. Biophys J 2004; 87: 4259-4270.

45 Matsui H, Verghese MW, Kesimer M, et al. Reduced three-dimensional motility in dehydrated airway mucus prevents neutrophil capture and killing bacteria on airway epithelial surfaces. J Immunol 2005; 175: 1090-1099.

46 Ma JT, Tang C, Kang L, et al. Cystic fibrosis sputum rheology correlates with both acute and longitudinal changes in lung function. Chest 2018; 154: 370-377.

47 Song Y, Salinas D, Nielson DW, et al. Hyperacidity of secreted fluid from submucosal glands in early cystic fibrosis. Am J Physiol Cell Physiol 2006; 290: C741-C749.

48 Pezzulo AA, Tang XX, Hoegger MJ, et al. Reduced airway surface $\mathrm{pH}$ impairs bacterial killing in the porcine cystic fibrosis lung. Nature 2012; 487: 109-113. 